\title{
CLIMATE RESPONSES
}

\section{Thermal bottlenecks in the life cycle define climate vulnerability of fish}

\author{
Flemming T. Dahlke ${ }^{1 *}$, Sylke Wohlrab ${ }^{1,2}$, Martin Butzin ${ }^{1}$, Hans-Otto Pörtner ${ }^{1,3 *}$
}

Species' vulnerability to climate change depends on the most temperature-sensitive life stages, but for major animal groups such as fish, life cycle bottlenecks are often not clearly defined. We used observational, experimental, and phylogenetic data to assess stage-specific thermal tolerance metrics for 694 marine and freshwater fish species from all climate zones. Our analysis shows that spawning adults and embryos consistently have narrower tolerance ranges than larvae and nonreproductive adults and are most vulnerable to climate warming. The sequence of stage-specific thermal tolerance corresponds with the oxygen-limitation hypothesis, suggesting a mechanistic link between ontogenetic changes in cardiorespiratory (aerobic) capacity and tolerance to temperature extremes. A logarithmic inverse correlation between the temperature dependence of physiological rates (development and oxygen consumption) and thermal tolerance range is proposed to reflect a fundamental, energetic trade-off in thermal adaptation. Scenario-based climate projections considering the most critical life stages (spawners and embryos) clearly identify the temperature requirements for reproduction as a critical bottleneck in the life cycle of fish. By 2100, depending on the Shared Socioeconomic Pathway (SSP) scenario followed, the percentages of species potentially affected by water temperatures exceeding their tolerance limit for reproduction range from $\sim 10 \%$ (SSP 1-1.9) to 60\% (SSP 5-8.5). Efforts to meet ambitious climate targets (SSP 1-1.9) could therefore benefit many fish species and people who depend on healthy fish stocks.

I he identification of biological patterns and underlying principles is fundamental for understanding and predicting ecological processes (I), including climate change effects (2). A central observation in this context is that aquatic ectothermic animals such as fish have specific temperature limits and tolerance ranges, which determine their latitudinal distribution limits and sensitivity to climate change (3). Mechanistic prin-

Fig. 1. Thermal tolerance differs between life stages, and thermal responsiveness is higher in stenothermal than in eurythermal organisms.

(A) Lower and upper temperature thresholds ( $T_{\min }$ and $\left.T_{\max }\right)$ are defined as ultimate temperature limits that relate to behavioral avoidance, impaired physiological functions, and mortality (22), thereby reflecting limits to the geographic distribution of species $(3,4)$. The specific temperature ranges $\left(T_{\text {range }}=T_{\max }-T_{\min }\right)$ of species are expected to differ between life stages according to changes in the relationship between oxygen demand and supply capacity (4). This hypothesis implies that from embryo to adult, aerobic capacity (and thus $T_{\text {range }}$ ) increases with the development of the cardiorespiratory system, but then declines again with increasing body size. During the spawning season, aerobic capacity and $T_{\text {range }}$ may decrease further as a result of additional energy requirements for the production of gametes and their biomass (2, 7). (B) Physiological rates (solid lines) are temperature-dependent and typically scale exponen-

ciples potentially related to this pattern include the temperature dependence of physiological rates and thus oxygen $\left(\mathrm{O}_{2}\right)$ demand $(4,5)$. Different cardiorespiratory capacities to sustain adequate $\mathrm{O}_{2}$ supply to tissues during temperature changes are suggested to explain why tolerance ranges are narrower for some fish species than for others (6). Thermal tolerance is also expected to change during the life cycle of species according to the development of aerobic capacities in relation to $\mathrm{O}_{2}$ demand $(2,7)$ (Fig. 1A). Specifically, tolerance ranges are hypothesized to widen from embryo to larval and adult stages after the development of cardiorespiratory organs $(7,8)$. During reproduction (spawning stage), tolerance ranges may narrow again as the result of a net decrease in aerobic capacity associated with additional energy and thus $\mathrm{O}_{2}$ demand for gamete production and biomass $(2,7)$. These principles are supported by empirical data for some wellstudied species such as Atlantic cod (Gadus morhua) (6), but it is currently unknown whether the proposed ontogenetic shift in thermal tolerance represents a globally consistent pattern.

Thermal adaption of species and life stages to local climatic conditions probably involves energetic optimizations and trade-offs $(9,10)$. Available data suggest that tolerance ranges are as narrow as possible to ensure survival under local conditions while minimizing costs for maintaining homeostasis over wide temperature ranges (6). Consequently, tolerance ranges are expected to reflect the magnitude of local temperature variability (11), with wider tolerance ranges in temperate regions relative to polar and tropical regions (12). Potential tradeoffs between energy efficiency and thermal tolerance may be linked to thermodynamic properties of metabolic processes (i.e., thermal responsiveness) $(9,13)$. Mechanistic theory predicts that "stenothermal" organisms with narrow temperature ranges display higher thermal responsiveness than more tolerant "eurythermal" organisms (13) (Fig. 1, B and C). In contrast, the concept of universal temperature dependence implies that there is no variation

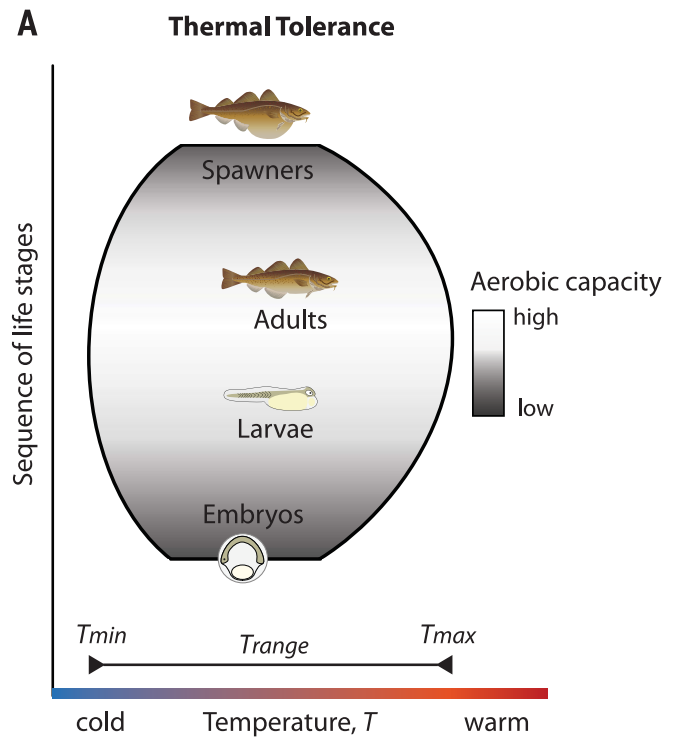

B Thermal Responsiveness
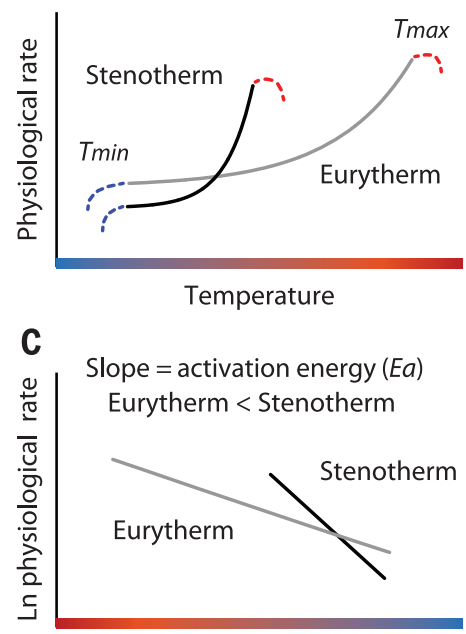

Inverse temperature $\left(1 /\left(\mathrm{k}^{*} T+273\right)\right.$ tially within $T_{\text {range }}$ (1). Lines with different slopes suggest that stenothermal species or life stages with narrow $T_{\text {range }}$ (black line) are more responsive (steeper slope) than eurythermal ones (gray line) (13). As a benefit, the energy demand of stenotherms may be lower than that of eurytherms (49). (C) Thermal responsiveness is quantified in Arrhenius form (log-transformed rate versus inverse absolute temperature) and is expressed as activation energy $\left(E_{a}\right)$ in electron volts (27). 
in thermal responsiveness among ectothermic species $[(14)$, but see $(15,16)]$.

Fish usually reproduce at certain times of the year (spawning seasons) and in certain places (spawning habitats) that provide suitable conditions for offspring survival $(17,18)$. Reproductive success is likely at risk under climate change when spawning habitat temperatures exceed the tolerance limit of the most sensitive life stage, forcing species to reproduce at different times and/or places (18). To date, mainly because of the scarcity of experimental data, potential life cycle bottlenecks in thermal tolerance are rarely considered in large-scale risk assessments (19-21), limiting our ability to determine whether climate mitigation targets are sufficient to sustain healthy fish stocks. Here, we address this limitation by combining experimental and observational data as well as phylogenetic data imputation (22) to generate a comprehensive set of stage-specific thermal tolerance metrics for 694 marine and freshwater fish species from all climate zones. With this dataset, we addressed the following hypotheses: (i) Upper and lower temperature limits $\left(T_{\max }, T_{\min }\right)$ and temperature ranges $\left(T_{\text {range }}=\right.$ $\left.T_{\max }-T_{\min }\right)$ differ consistently between life stages (Fig. 1A). (ii) Thermal responsiveness is higher in stenothermal organisms than in eurythermal ones (Fig. 1B). Furthermore, stagespecific tolerance limits were used to assess climatic risks under different Shared Socioeconomic Pathway (SSP) scenarios of global change developed during the sixth phase of the Coupled Model Intercomparison Project (CMIP6) (23).

\section{Thermal tolerance depends on phylogeny, geographic origin, and ontogeny}

We collated empirical thermal tolerance data of four life stages: egg stage ("embryos"), pre-metamorphosis stage ("larvae"), postmetamorphosis stage ("adults"), and reproductive stage ("spawners"). Data on embryos, larvae, and adults are experimental estimates of physiological ultimate temperature limits (22). Because experimental data for spawners are extremely scarce, in situ observations (e.g., fisheries monitoring and tagging data) of behavioral temperature limits were considered for this life stage. A direct comparison revealed no significant difference between experimental and observational $T_{\max }$ data of spawners (two-sided paired $t$ test, $P=0.189, n=15$ species; fig. $\mathrm{S} 1$ and table $\mathrm{S} 1)$. Temperature ranges $\left(T_{\text {range }}\right)$ were estimated as the difference between $T_{\max }$ and $T_{\text {min }}$. Midpoint temperatures $\left(T_{\text {mid }}\right)$ are

\footnotetext{
${ }^{1}$ Alfred Wegener Institute, Helmholtz Centre for Polar and Marine Research, 27570 Bremerhaven, Germany. ${ }^{2}$ Helmholtz Institute for Functional Marine Biodiversity, 26129 Oldenburg, Germany. ${ }^{3}$ University of Bremen, 28359 Bremen, Germany. *Corresponding author. Email: flemming.dahlke@gmx.de (F.T.D.); hans.poertner@awi.de (H.-O.P.)
}
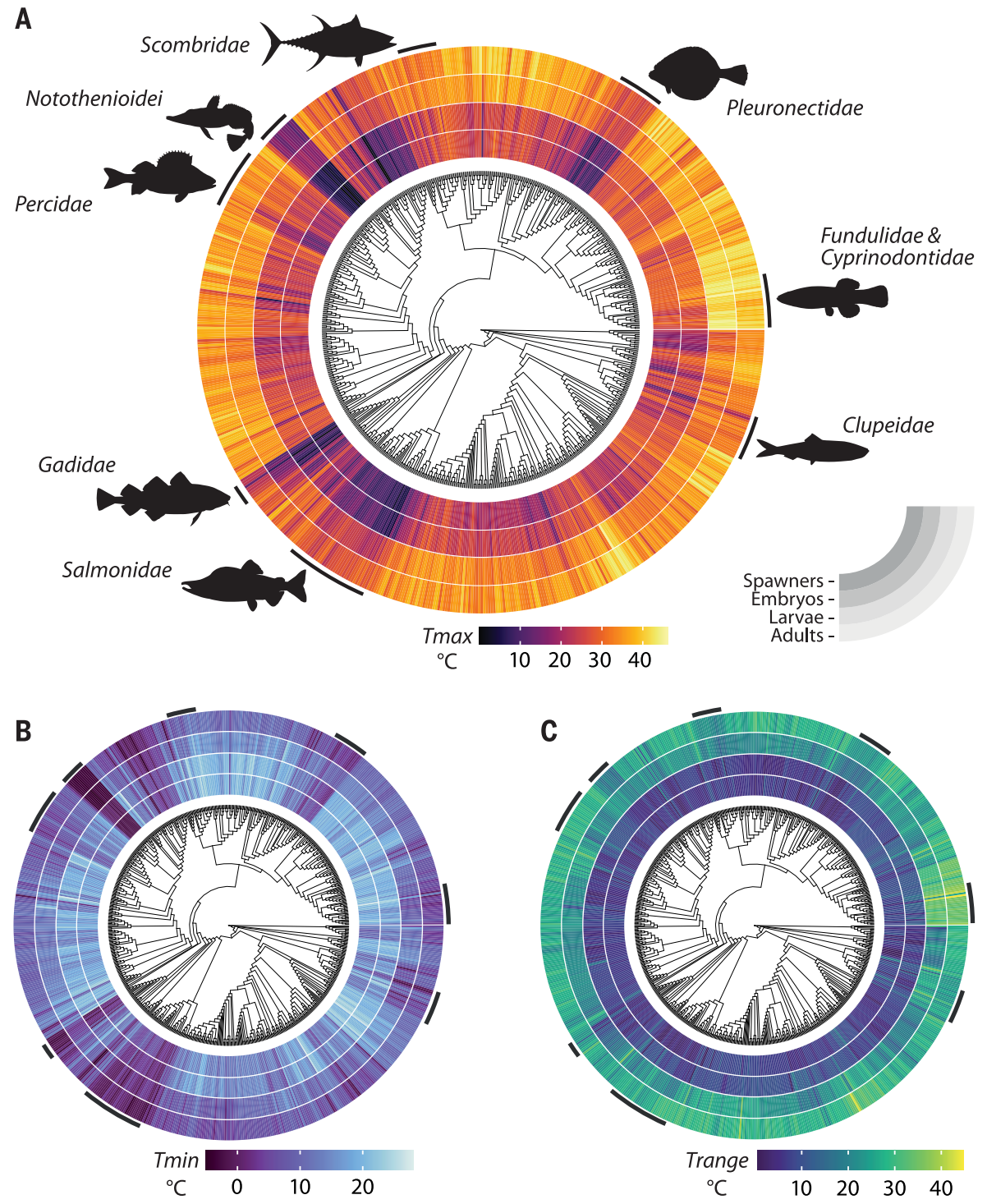

Fig. 2. Phylogenetic and ontogenetic patterns in thermal tolerance of fish. (A to $\mathbf{C})$ Circular chronograms show upper temperature limits $\left(T_{\max }\right)(A)$, lower temperature limits $\left(T_{\min }\right)(B)$, and thermal tolerance ranges $\left(T_{\text {range }}\right)(C)$ of species and their life stages from inside to outside: spawners, embryos, larvae, and adults. Prominent taxonomic groups as well as particularly warm-eurythermal (Fundulidae and Cyprinodontidae) and cold-stenothermal groups (Notothenioidei) are highlighted.

either observed temperature optima or arithmetic means of $T_{\min }$ and $T_{\max }$. For 694 of 777 species, we obtained at least one empirical thermal tolerance metric $\left(T_{\max }, T_{\min }\right.$, or $\left.T_{\text {mid }}\right)$ as well as time-calibrated phylogeny from the Fish Tree of Life (24). Phylogenetic imputation (22) was then used to estimate missing $T_{\max }$ and $T_{\min }$ values. We confirmed phylogenetic niche conservatism (i.e., similar trait values among closely related species) as a prerequisite for reliable data imputation (25) a priori, based on phylogenetic signal indices (Pagel's $\lambda$, $P<0.001$; table S2). Precision of imputed data was assessed on the basis of correlation analysis and variance estimation (fig. S2 and table S3). Ontogenetic differences in thermal toler- ance were assessed using generalized additive models (GAMs), taking into account variation related to geographic origin (latitude and marine versus freshwater) as well as uncertainty related to data imputation by including the inverse of estimated variances as weights.

The completed dataset (26) reveals phylogenetic and ontogenetic patterns in thermal tolerance of fish (Fig. 2). In addition to existing evidence for niche conservatism in adult fish (20), we find that phylogenetic clustering of temperature limits ( $T_{\max }$ and $T_{\min }$; Fig. $2, \mathrm{~A}$ and B) and tolerance ranges ( $T_{\text {range }} ;$ Fig. $\left.2 \mathrm{C}\right)$ is consistent across life stages (table S2). In line with expected ontogenetic shifts in aerobic capacity (2), we find that $T_{\text {range }}$ is narrower 
Fig. 3. Ontogenetic changes in thermal tolerance are consistent across latitudes. (A to C) Upper temperature limits $\left(T_{\max }\right)(A)$, lower temperature limits $\left(T_{\min }\right)$ (B), and thermal tolerance ranges $\left(T_{\text {range }}=\right.$ $\left.T_{\max }-T_{\min }\right)(\mathrm{C})$ of spawners (black), embryos (blue), larvae (orange), and adults (red) as a function of absolute latitude. Regression fits (solid lines) with 95\% Cls (colored shadings) are based on generalized additive models (GAM, $P<0.0001 ; n=698$ to 735 for each life stage), accounting for uncertainty related to phylogenetic data imputation (22). Only the correlation between latitude and $T_{\text {range }}$ of embryos (C) is not significant ( $P>0.05)$. (D to
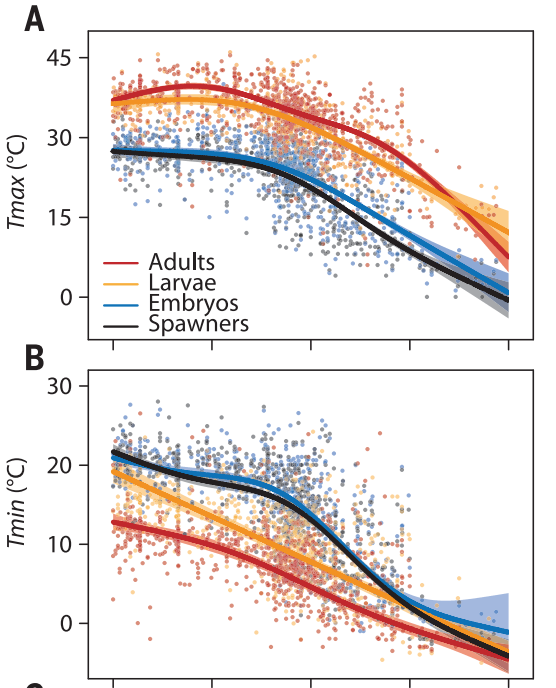

C

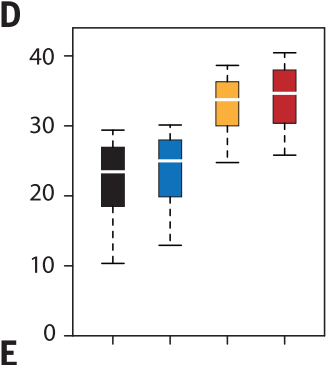

E
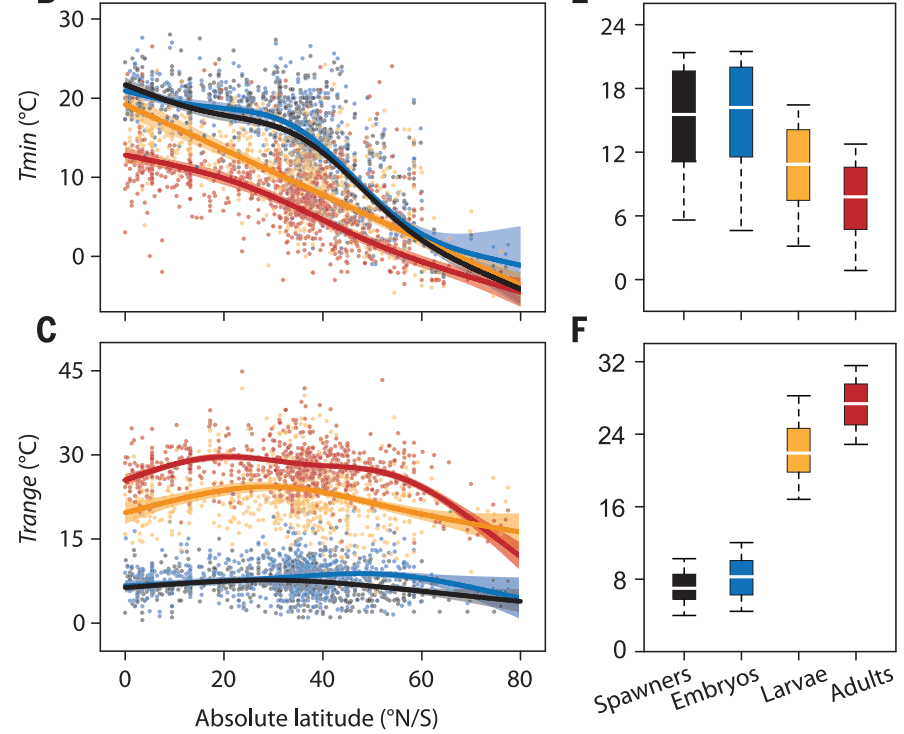

$\mathbf{F}$

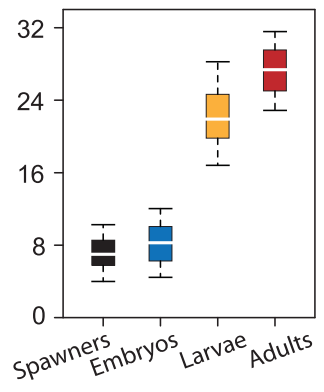

F) Corresponding to (A) to (C), box plots indicate differences in $T_{\max }, T_{\min }$, and $T_{\text {range }}$ between life stages. Boxes and whiskers show 25th to 75th and 10th to 90th percentiles, respectively; white lines indicate the median. When accounting for geographic variation (latitude and marine versus freshwater; table S4), life stages differ significantly in terms of $T_{\max }, T_{\min }$, and $T_{\text {range }}$ (two-sided pairwise comparisons with Tukey correction, all $P<0.05$ ).

for spawners and embryos than for larvae and adults (GAM, $P<0.0001$; Fig. 2 C and table S4). Ontogenetic differences in thermal tolerance metrics are also consistent across latitudes (Fig. 3, A to C) and aquatic realms (i.e., marine and freshwater) (fig. S3). In support of the climate variability hypothesis (11), $T_{\text {range }}$ tends to decrease toward high and low latitudes (Fig. 3C and table S4), with the most stenothermal species found in the Antarctic Ocean (Antarctic icefishes, Notothenioidei) and the most eurythermal ones in temperate freshwater systems (killifishes, Fundulidae) (Fig. 2A). Thermal tolerance ranges of freshwater species and their life stages are on average $\sim 1^{\circ} \mathrm{C}$ wider than for marine species (GAM, $P<0.0001$; fig. S3), probably reflecting higher temperature variability in lakes and rivers than in oceans. When accounting for geographic variation in thermal tolerance metrics, mean $T_{\text {range }}$ values $[ \pm 95 \%$ confidence intervals (CIs)] increase by more than $20^{\circ} \mathrm{C}$ from spawners $\left(7.2^{\circ} \pm 0.3^{\circ} \mathrm{C}\right)$ and embryos $\left(8.4^{\circ} \pm 0.4^{\circ} \mathrm{C}\right)$ to larvae $\left(22.3^{\circ} \pm 0.7^{\circ} \mathrm{C}\right)$ and adults $\left(27.5^{\circ} \pm 0.4^{\circ} \mathrm{C}\right)$ (Fig. $\left.3 \mathrm{~F}\right)$. These results clearly identify the temperature requirements of spawners and embryos as critical bottlenecks in the life cycle of fish.

\section{Thermal responsiveness is inversely correlated with thermal tolerance}

Thermal responsiveness and its correlation with thermal tolerance ( $T_{\text {range }}$ ) were assessed on the basis of the temperature dependence of development rate (DR) and oxygen consumption rate $\left(\mathrm{MO}_{2}\right)$ of embryos (DR and $\mathrm{MO}_{2}, 83$ species), larvae $\left(\mathrm{MO}_{2}, 16\right.$ species $)$ and adults $\left(\mathrm{MO}_{2}\right.$, 54 species) (26). Direct comparison of the temperature dependences of $\mathrm{DR}$ and $\mathrm{MO}_{2}$ revealed no systematic difference between them (fig. S4). Only experimental data measured under controlled, noncritical temperature conditions were used (22). Individual responses (Fig. 4A) were evaluated using the Boltzmann-Arrhenius function (27), in which the scaling of physiological rates $(R)$ with temperature $(T)$ is

$$
R=R_{0} \exp \left(\frac{-E_{\mathrm{a}}}{k T}\right)
$$

Thermal responsiveness is given by the value of $-E_{\mathrm{a}}$ in electron volts (eV, positivized hereafter), which is the activation energy of the rate-limiting biochemical (metabolic) process, $R_{0}$ is an organism-specific coefficient, and $k$ is Boltzmann's constant. Analyses of ontogenetic differences in $E_{\mathrm{a}}$ (linear mixed-effect model, LMM) and the correlation between $E_{\text {a }}$ and $T_{\text {range }}$ (generalized additive mixed-effect model, GAMM) accounted for phylogenetic nonindependence.

Thermal responsiveness of embryos is on average $24 \%$ higher than in larvae and adults (LMM, $P<0.0001$; Fig. 4B). Thermal responsiveness of embryos (mean $E_{\mathrm{a}}, 0.87 \mathrm{eV} ; 95 \% \mathrm{CI}$, 0.83 to $0.91 \mathrm{eV}$ ) also exceeds the range predicted by the universal temperature dependence concept (0.6 to $0.7 \mathrm{eV}$ ) (14). The correlation between $E_{\mathrm{a}}$ and $T_{\text {range }}$ (GAMM, $P<0.0001$; Fig. 4C) supports the hypothesis that the metabolism of stenotherms is more responsive to temperature changes than that of eurytherms $(4,13)$. The correlation between $E_{\mathrm{a}}$ and $T_{\text {range }}$ is also consistent within life stages (embryos and adults) and within individual species such as Atlantic cod (fig. S5). These results suggest that temperature-rate responses do not strictly conform to statistical thermodynamics (14) but instead may reflect an outcome of energetic optimizations (reduction of costs) and tradeoffs related to lifestyle and ontogeny (10). Biophysical models using a generalized $E_{\mathrm{a}}$ of 0.6 to $0.7 \mathrm{eV}(14,28)$ rather than the actual responsiveness of fish species or life stages may therefore lead to imprecise projections of ecological processes. For example, a scenario that assumes $3^{\circ} \mathrm{C}$ warming and an embryonic $E_{\mathrm{a}}$ of $0.65 \mathrm{eV}$ would underestimate the change in development time of Atlantic cod embryos $\left(E_{\mathrm{a}}=0.92 \mathrm{eV}\right)(29)$ and associated probability functions (e.g., predation mortality) by $\sim 80 \%$ (fig. S6).

\section{Safety margins of critical life stages define species' vulnerability to warming}

The vulnerability of species to climate warming is often assessed according to the difference between the upper thermal tolerance limit $\left(T_{\max }\right)$ of adult life stages and the maximum habitat temperature during summer-a metric called the thermal safety margin (TSM). However, the tolerance of adults to summer heat is potentially less critical for the persistence of species than the ultimate temperature limit for reproduction, which we assessed on the basis of $T_{\max }$ of spawners and embryos. Specifically, we compared current and future TSMs of adults (the difference between $T_{\max }$ and the mean temperature of the warmest summer month) with the TSMs of spawners and embryos, estimated as the difference between their $T_{\max }$ and the mean temperature of the coldest month during the species-specific spawning season [mainly spring or monsoon time (22)]. In this way, future TSMs of spawners and embryos indicate whether climate change will affect the ability of species to reproduce at preferred times and locations. Seasonal habitat temperatures of adults, spawners, and embryos [630 species (26)] are spatial averages according to distribution records, depth preferences, 
Fig. 4. Thermal responsiveness is higher in stenothermal life stages. (A) Exponential temperature responses of embryos (blue), larvae (orange), and adults (red) based on Eq. 1. Thermal responsiveness is indicated by the slope of individual responses (thin lines). Thick lines indicate the median responsiveness of different life stages. Normalization (response values estimated at any temperature divided by their value at $15^{\circ} \mathrm{C}$ ) was done for illustrative purposes. (B) Thermal responsiveness expressed as Arrhenius activation energy ( $E_{\mathrm{a}}$ in electron volts, eV). Box plots (as in Fig. 3) with different letters indicate significant differences between life stages (two-sided pairwise comparisons with Tukey correction, $P<0.05$ ).

(C) Correlation between thermal responsiveness and thermal tolerance of different life stages (colored symbols). The regression fit (line with 95\% Cls as shading) accounts for phylogenetic nonindependence (generalized additive mixed-effect model, GAMM).

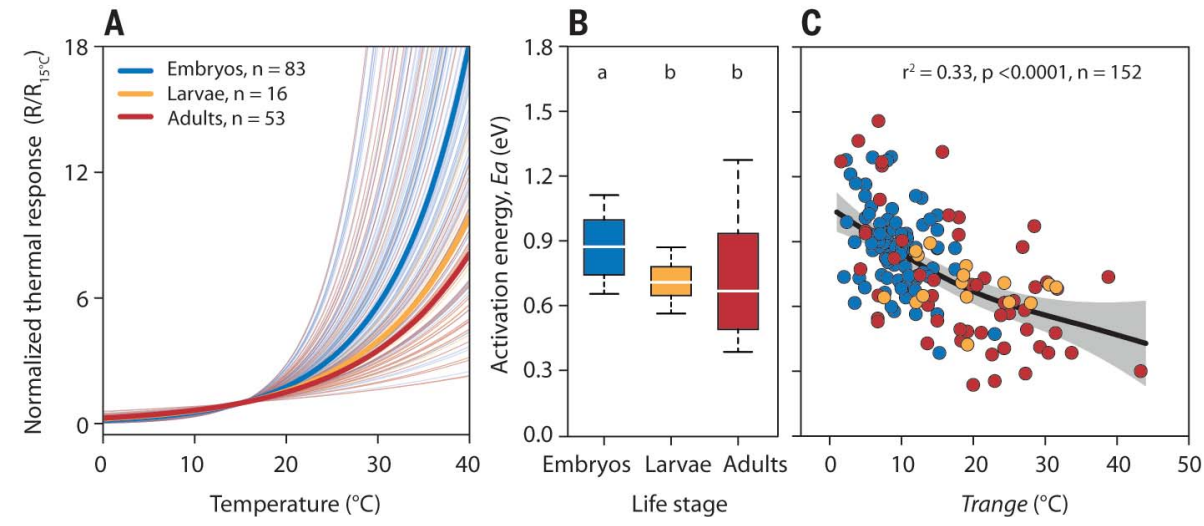

Fig. 5. Smaller safety margins of spawners and embryos versus adults. (A) Thermal safety margins (TSMs) of spawners (black), embryos (blue), and adults (red) based on recent habitat (water) temperatures (1981 to 2000) as a function of absolute latitude. Regression fits (colored lines) with 95\% Cls as shadings are based on generalized additive models (GAM, $P<0.0001$; $n=543$ for spawners, 554 for embryos, 580 for adults; 630 species in total), taking into account uncertainty related to phylogenetic data imputation (24). (B) Corresponding to (A), box plots (as in Fig. 3) with different letters indicate significant differences between TSMs of different life stages (two-sided pairwise comparisons with Tukey correction, $P<0.05$ ).
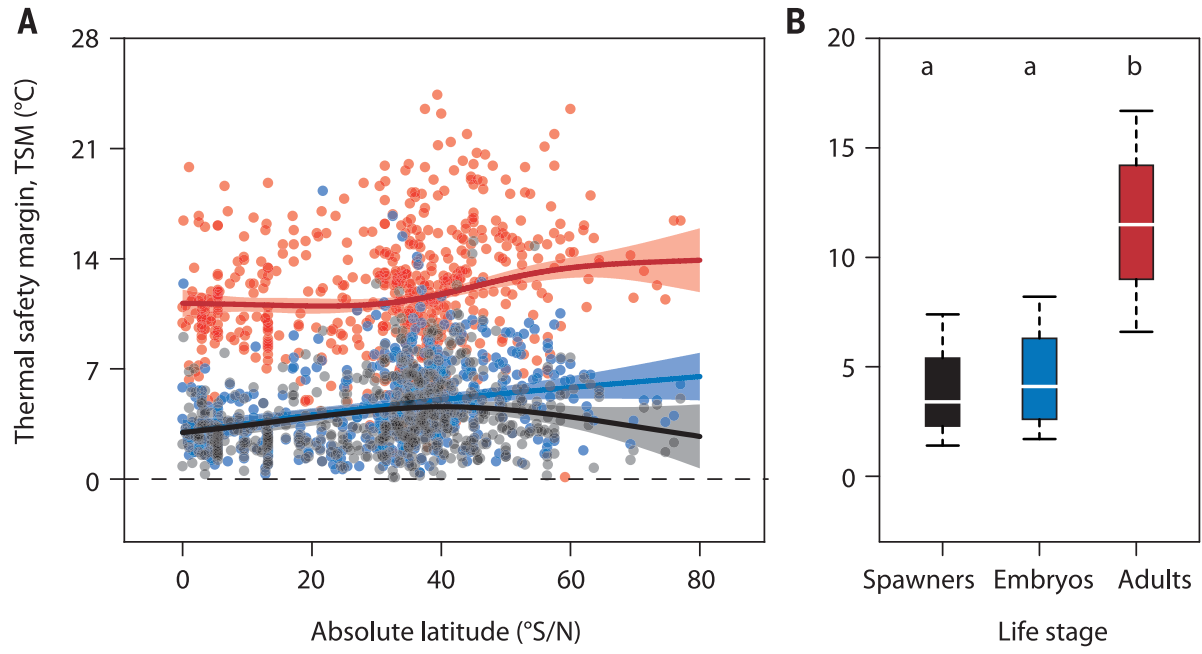

marine and freshwater species from different latitudes may already experience critically warm temperature conditions for reproduction. Although we note that the horizontal resolution of observed and simulated habitat temperatures $\left(1^{\circ} \times 1^{\circ}\right)$ is not always sufficient to capture specific microclimates, the cutoff threshold for considering a species at risk from future warming is set to TSM $\leq 0.0^{\circ} \mathrm{C}$.

Projected changes in TSMs of the most sensitive life stage (67\% spawners and $33 \%$ embryos) differ considerably between species and emission scenarios (Fig. 6 and figs. S7 to S9). By the end of this century, more than $60 \%$ (median of five models) of marine and freshwater species considered in this study could be confronted with water temperatures exceeding tolerance limits in their current habitat (TSM $\leq$ $0.0^{\circ} \mathrm{C}$ ) if emissions continue to rise unabated (SSP 5-8.5; Fig. 6B). TSMs below zero indicate that reproduction at preferred seasons and locations is no longer possible, forcing species to adapt or shift their spawning activity into cooler seasons or regions to avoid extinction. Emission pathways consistent with current political commitments (SSP 4-6.0) would still threaten more than one-third of the marine and freshwater species under consideration (Fig. 6B). A positive outlook is that the percentage of species at risk could be reduced to 10 to $15 \%$ if global warming is limited to $1.5^{\circ} \mathrm{C}$ (SSP 1-1.9; Fig. 6B), in line with the Paris Agreement (30). For comparison, when considering adult TSMs only, the fraction of species below the cutoff threshold is below 5\% under SSP 5-8.5 (Fig. 6B). Accordingly, although assessments based on adult temperature limits or proxies thereof (e.g., species distribution records) can provide important information on the geographical distribution of climatic vulnerability (19-21), such analyses miss the most sensitive life stages and are likely to underestimate impact risks at the species level.

\section{Discussion}

This study identifies spawners and embryos as the most temperature-sensitive stages in the life cycle of fish. The observed ontogenetic shift in thermal tolerance is consistent with
TSMs of spawners and embryos (TSM between $1^{\circ}$ and $0^{\circ} \mathrm{C}, n=37$; Fig. 5) indicate that some 
A

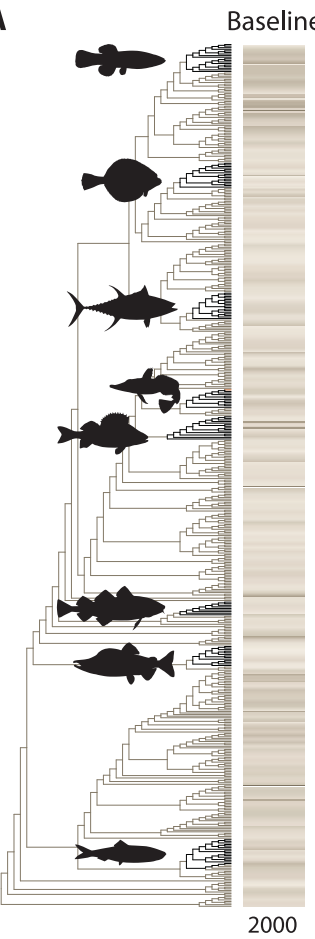

SSP1-1.9

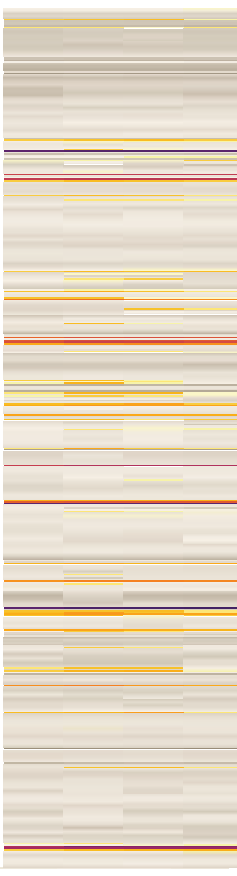

2040206020802100
SSP4-6.0
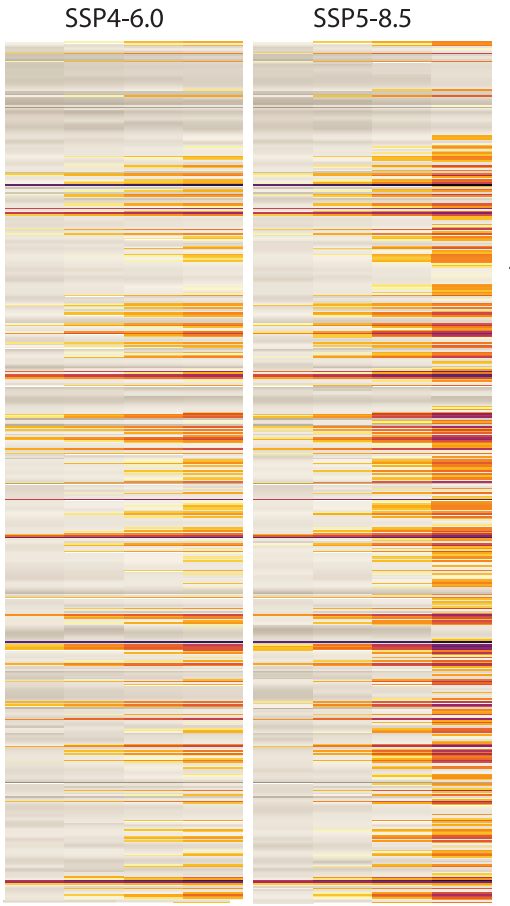

$\operatorname{TSM}\left({ }^{\circ} \mathrm{C}\right)$

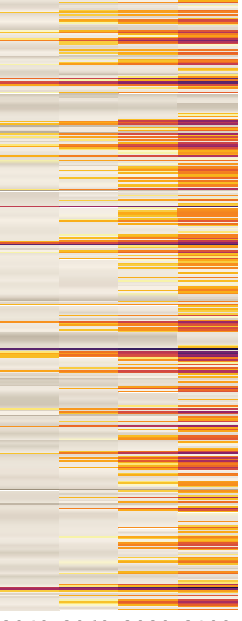

B

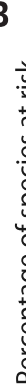

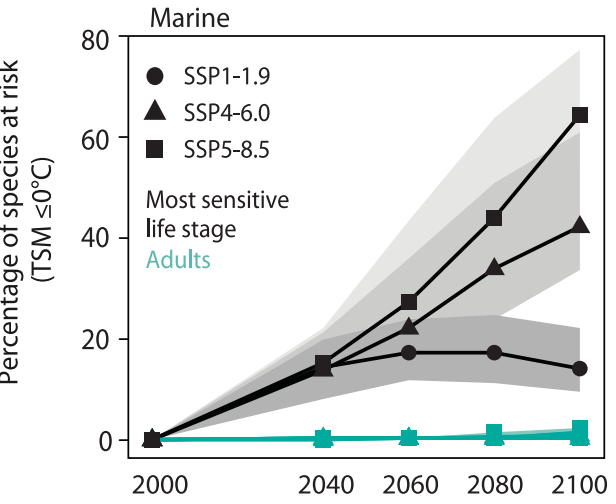

\begin{abstract}
80 Marine
\end{abstract}
Freshwater

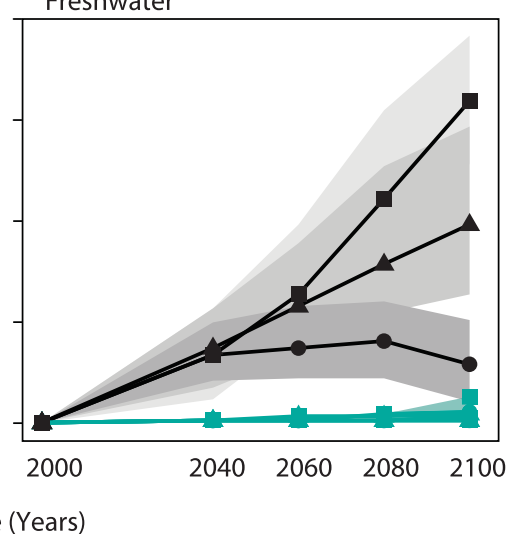

Fig. 6. Shrinking safety margins of critical life stages put many fish species at risk in their current habitat. (A) Cladogram for investigated fish species with tips colored according to estimated TSMs for recent conditions (1981 to 2000), and future Shared Socioeconomic Pathway (SSP) scenarios considering the TSM of the most critical life stages ( 67\% spawners, $\sim 33 \%$ embryos) in their respective habitats. SSP scenarios were developed during the sixth phase of the Coupled Model Intercomparison Project (CMIP6), representing low (SSP 1-1.9), intermediate (SSP 4-6.0), and high (SSP 5-8.5) radiative forcing pathways (23). (B) Median percentage of marine $(n=367)$ and freshwater species $(n=263)$ with TSMs less than or equal to $0.0^{\circ} \mathrm{C}$. Black lines consider TSMs of the most sensitive life stage; green lines indicate adult TSMs $\leq 0.0^{\circ} \mathrm{C}$. Gray shadings denote the range between lower and upper percentile bounds of the climate model ensemble scenarios.

previous analyses of smaller datasets $(17,31)$ and corresponds to the concept of oxygen- and capacity-limited thermal tolerance (4), suggesting that ontogenetic changes in aerobic capacity lead to corresponding changes in upper and lower temperature limits (2). Increases in aerobic capacity from egg to adult follow the development of the cardiorespiratory system (heart and gills), which facilitates effective $\mathrm{O}_{2}$ supply to tissues and thus improves tolerance to temperature extremes (8). In addition, the development of homeostasis functions (e.g., ion regulation) and repair mechanisms, including heat shock responses, may contribute to an increase in tolerance to extreme temperatures from egg to adult $(32,33)$. Immature aerobic and homeostatic capacities of fish embryos and larvae also explain their sensitivity to other environmental factors such as hypoxia, salinity stress, and $\mathrm{CO}_{2}$-driven acidification $(29,34)$. When adults become sexually mature, additional metabolic loads for gamete production [often $>20 \%$ of body mass (35)] are expected to cause a concomitant decrease in thermal tolerance due to warming-induced loss in aerobic capacity $(2,7)$. Hypoallometric growth of aerobic capacity relative to body mass may also lead to a narrowing of tolerance ranges and a shift toward lower optimal temperatures in large, nonreproducing adults, as suggested by experiments with Atlantic cod (36) and field observations in eelpout (Zoarces viviparus) (5). Indirect evidence of reduced aerobic capacity of spawners relative to nonreproductive adults comes from experiments indicating increased sensitivity to hypoxia at the final stage of gonadal development (37). In addition to oxygen limitation, temperature stress can directly impair gonadal development by affecting the production and release of sex hormones (38). Narrow tolerance ranges of spawners may therefore reflect a combination of thermal constraints on aerobic metabolism and endocrine processes related to gametogenesis. The pattern of stage-specific thermal tolerance demonstrated in this study may also apply to other ectothermic animals (39), although oxygen limitation as a causal principle may be more relevant for aquatic versus terrestrial organisms, owing to the much lower concentration and diffusivity of oxygen in water.

The higher thermal responsiveness of stenothermal species relative to eurythermal species and life stages implies a mechanistic link between physiological thermodynamics and organismal thermal tolerance in fish $(10,13)$. This prediction is based on the idea that elevated activation energies (kinetic barriers) of rate-limiting reactions in the citric acid cycle constrain metabolic flux and cost and promote resource efficiency (13). However, as relatively small temperature changes have a marked impact on metabolic and developmental rates, such an energetic optimization associated with tolerance to a restricted temperature range (i.e., stenothermality) is beneficial only when environmental temperature variability is low (Fig. 4C). Eurythermal organisms are thermally less responsive and have wider tolerance ranges, which promote an active lifestyle in habitats with daily, seasonal, and/or vertical temperature gradients and variability. For instance, low responsiveness allows relatively constant levels of performance and thus foraging ability in summer and winter as well as across depthrelated temperature gradients. The trade-off in this case may involve elevated baseline energy turnover, as seen in many eurythermal species with an active pelagic lifestyle (40). Accordingly, in contrast to the concept of universal temperature dependence (14), we argue that thermal responsiveness is adaptive and shaped to meet environmental conditions and ecological 
requirements (9). Building on the indirect evidence presented here (correlation between $E_{\mathrm{a}}$ and $T_{\text {range }} ;$ Fig. $4 \mathrm{C}$ ), further experimental work and analyses that take into account potentially relevant traits (e.g., body mass, activity, trophic level) are necessary to confirm a general relationship among energy efficiency, metabolic thermal responsiveness, and thermal tolerance range.

Narrow thermal safety margins of spawners and embryos indicate that the temperature requirements for reproduction define the climate change vulnerability of fish. For many species, the highest warming trajectory (SSP 5-8.5) represents a major threat, as water temperature may exceed their current tolerance limit for reproduction (Fig. 6). Coping with climate change would be achieved through changes in thermal tolerance [through acclimatization of individuals, or through evolutionary adaptation across generations (41)] and by shifting the timing and/or location of spawning to cooler seasons or regions [niche tracking $(42,43)]$. However, adaptation over generations is probably too slow to cope with major anthropogenic change $(44,45)$. In addition, shifts in spawning times and locations can be problematic and in some cases impossible, depending on species' reproductive strategy and geographical distribution (18). For instance, to provide offspring with suitable feeding conditions, spawning times are usually synchronized with seasonal peaks in plankton productivity (46), especially outside the tropics (18). Moreover, spawning locations may provide essential substrates for egg deposition and hydrographic features that ensure dispersal of pelagic eggs and larvae toward suitable nursery habitats (47). This means that despite more suitable temperature conditions, alternative spawning seasons and locations may not necessarily meet the ecological requirements for successful reproduction (18). Relative to most marine species, freshwater fishes are less flexible in terms of niche tracking because of geographic barriers between habitats and anthropogenic habitat degradation (e.g., dams, hydroelectric power plants, and pollution) (20).

Note that quantitative risk assessments for individual species or populations (e.g., changes in abundance and productivity) may require not only information on sublethal temperature thresholds constraining functional scope (e.g., indicated by reduced growth performance) (5), but also more detailed data on acclimatization and adaptation potential, spawning ecology, population structure, habitat connectivity, and microclimatic conditions than are available for most species considered in this study. Furthermore, our qualitative risk assessment is probably conservative because exposure to additional climate change factors such as deoxygenation, acidification, and temperature extremes during stochastic heat waves (30) is not considered.

\section{Conclusion}

Phylogenetic, geographic, and ontogenetic patterns in thermal physiology revealed in this study suggest that many fish species face greater risks due to global warming than previously expected. Narrow temperature ranges for reproduction in relation to future warming scenarios underscore the urgency to investigate the adaptive potential of species and populations while taking measures to protect existing and alternative (spawning) habitats from human impacts (48). Very clearly, many fish species and people who depend on healthy fish stocks would benefit from intensified efforts to stabilize global warming at $1.5^{\circ} \mathrm{C}$ or even less.

\section{REFERENCES AND NOTES}

1. A. Clarke, Principles of Thermal Ecology: Temperature, Energy and Life (Oxford Univ. Press, 2017).

2. H.-O. Pörtner, A. P. Farrell, Science 322, 690-692 (2008).

3. J. M. Sunday, A. E. Bates, N. K. Dulvy, Nat. Clim. Change 2, 686-690 (2012).

4. H.-O. Pörtner, Comp. Biochem. Physiol. A 132, 739-761 (2002).

5. H.-O. Pörtner, R. Knust, Science 315, 95-97 (2007).

6. H.-O. Pörtner, C. Bock, F. C. Mark, J. Exp. Biol. 220, 2685-2696 (2017)

7. H.-O. Pörtner, M. A. Peck, J. Fish Biol. 77, 1745-1779 (2010).

8. P. J. Rombough, in Fish Physiology, vol. 11 (Elsevier, 1988), pp. 59-161.

9. A. Clarke, Trends Ecol. Evol. 18, 573-581 (2003).

10. H.-0. Pörtner et al., Physiol. Biochem. Zool. 79, 295-313 (2006)

11. D. H. Janzen, Am. Nat. 101, 233-249 (1967).

12. J. Sunday et al., Philos. Trans. R. Soc. London Ser. B 374 , 20190036 (2019).

13. H.-O. Pörtner, M. Lucassen, D. Storch, Fish Physiol. 22, 79-154 (2005).

14. J. H. Brown, J. F. Gillooly, A. P. Allen, V. M. Savage, G. B. West, Ecology 85, 1771-1789 (2004)

15. A. I. Dell, S. Pawar, V. M. Savage, Proc. Natl. Acad. Sci. U.S.A. 108, 10591-10596 (2011).

16. T. P. Smith et al., Nat. Commun. 10, 5124 (2019).

17. J. Brett, Q. Rev. Biol. 31, 75-87 (1956)

18. L. Ciannelli, K. Bailey, E. M. Olsen, ICES J. Mar. Sci. 72, 285-296 (2015).

19. M. L. Pinsky, A. M. Eikeset, D. J. McCauley, J. L. Payne, J. M. Sunday, Nature 569, 108-111 (2019).

20. L. Comte, J. D. OIden, Nat. Clim. Change 7, 718-722 (2017).

21. C. H. Trisos, C. Merow, A. L. Pigot, Nature 580, 496-501 (2020).

22. See supplementary materials.

23. M. Gidden et al., Geosci. Model Dev. 12, 1443-1475 (2019).

24. D. L. Rabosky et al., Nature 559, 392-395 (2018).

25. E. W. Goolsby, J. Bruggeman, C. Ané, Methods Ecol. Evol. 8, 22-27 (2017)

26. See the acknowledgments (data and materials availability).

27. R. M. Sibly, J. H. Brown, A. Kodric-Brown, Metabolic Ecology: A Scaling Approach (Wiley, 2012).

28. M. E. Dillon, G. Wang, R. B. Huey, Nature 467, 704-706 (2010).

29. F. T. Dahlke et al., Sci. Adv. 4, eaas8821 (2018).

30. 0. Hoegh-Guldberg et al., Science 365, eaaw6974 (2019)

31. P. J. Rombough, in Seminar Series-Society for Experimental Biology, vol. 61 (Cambridge Univ. Press, 1997), pp. 177-224.

32. A. Hamdoun, D. Epel, Proc. Natl. Acad. Sci. U.S.A. 104, 1745-1750 (2007)
33. F. Dahlke et al., J. Exp. Biol. 223, jeb212589 (2020)

34. R. Przeslawski, M. Byrne, C. Mellin, Glob. Change Biol. 21 2122-2140 (2015)

35. D. R. Barneche, D. R. Robertson, C. R. White, D. J. Marshall, Science 360, 642-645 (2018)

36. B. Björnsson, A. Steinarsson, Can. J. Fish. Aquat. Sci. 59 494-502 (2002)

37. R. S. Wu, in Fish Physiology, vol. 27 (Elsevier, 2009), pp. $79-141$.

38. N. W. Pankhurst, P. L. Munday, Mar. Freshw. Res. 62 1015-1026 (2011).

39. B. S. Walsh et al., Trends Ecol. Evol. 34, 249-259 (2019).

40. B. A. Seibel, J. C. Drazen, Philos. Trans. R. Soc. London Ser. B 362, 2061-2078 (2007).

41. M. J. J. Angilletta, Thermal Adaptation: A Theoretical and Empirical Synthesis (Oxford Univ. Press, 2009).

42. M. L. Pinsky, B. Worm, M. J. Fogarty, J. L. Sarmiento, S. A. Levin, Science 341, 1239-1242 (2013).

43. A. Bruge, P. Alvarez, A. Fontán, U. Cotano, G. Chust, Front. Mar. Sci. 3, 86 (2016).

44. M. Byrne, S. A. Foo, P. M. Ross, H. M. Putnam, Glob. Change Biol. 26, 80-102 (2020)

45. H.-O. Pörtner et al., Ocean systems. In Climate Change 2014: Impacts, Adaptation, and Vulnerability. Part A: Global and Sectoral Aspects. Contribution of Working Group II to the Fifth Assessment Report of the Intergovernmental Panel on Climate Change (Cambridge Univ. Press, 2014).

46. A. B. Neuheimer, B. R. MacKenzie, M. R. Payne, Sci. Adv. 4 eaar4349 (2018).

47. R. J. Wootton, C. Smith, Reproductive Biology of Teleost Fishes (Wiley, 2014)

48. C. M. Duarte et al., Nature 580, 39-51 (2020).

49. H.-O. Pörtner et al., Clim. Res. 37, 253-270 (2008)

\section{ACKNOWLEDGMENTS}

We thank D. Storch for constructive comments on an earlier version of this manuscript. The Earth System Grid Federation is acknowledged for providing access to CMIP6 climate model output. Funding: Supported by the research project METAFISCH of the German Federal Ministry of Education and Research (BMBF grant FZKO1LS1604A to H.-O.P. and F.T.D.) and by the Deutsche Forschungsgemeinschaft (Po 278/16-1 and -2) as part of the Research Unit Tersane (FO 2332). S.W. was funded through HIFMB based on the collaboration between the Alfred Wegener Institute, Helmholtz Centre for Polar and Marine Research, and the Carl von Ossietzky University Oldenburg, initially funded by the Ministry for Science and Culture of Lower Saxony and the Volkswagen Foundation through the Niedersächsisches Vorab grant program (grant ZN3285) Author contributions: H.-O.P. and F.T.D. conceived the research idea; F.T.D. collated thermal tolerance and thermal responsiveness data and coordinated all analyses and visualizations; S.W. compiled distribution data and conducted phylogenetic imputation of thermal tolerance data, including visualization; M.B. compiled and analyzed climate model data, including the calculation of habitat temperatures; and F.T.D. drafted the manuscript. All authors wrote and edited the manuscript. Competing interests: The authors declare that they have no competing interests. Data and materials availability: All data needed to evaluate the conclusions in the paper are present in the paper and/or the supplementary materials and data files. The raw data supporting the findings of this study are available from PANGAEA, a member of the ICSU World Data System, at https://doi.org/10.1594/PANGAEA.917796.

\section{SUPPLEMENTARY MATERIALS}

science.sciencemag.org/content/369/6499/65/suppl/DC1 Materials and Methods

Figs. S1 to S10

Tables S1 to S6

References (50-127)

View/request a protocol for this paper from Bio-protocol.

4 September 2019; accepted 14 May 2020

10.1126/science.aaz3658 


\section{Science}

\section{Thermal bottlenecks in the life cycle define climate vulnerability of fish}

Flemming T. Dahlke, Sylke Wohlrab, Martin Butzin and Hans-Otto Pörtner

Science 369 (6499), 65-70.

DOI: $10.1126 /$ science.aaz3658

\section{Some cope better than others}

Increasingly, research is revealing how organisms may, or may not, adapt to a changing climate. Understanding the limitations placed by a species's physiology can help to determine whether it has an immediate potential to deal with rapid change. Many studies have looked at physiological tolerance to climate change in fishes, with results indicating a range of responses. Dahlke et al. conducted a meta-analysis to explore how life stage may influence a species's ability to tolerate temperature change (see the Perspective by Sunday). They found that embryos and breeding adult fishes are much more susceptible to temperature change than those in other life stages and that this factor must therefore be considered in evaluations of susceptibility.

Science, this issue p. 65 ; see also p. 35

ARTICLE TOOLS

SUPPLEMENTARY MATERIALS

RELATED

CONTENT

REFERENCES

PERMISSIONS http://science.sciencemag.org/content/369/6499/65

http://science.sciencemag.org/content/suppl/2020/07/01/369.6499.65.DC1

http://science.sciencemag.org/content/sci/369/6499/35.full

This article cites 102 articles, 13 of which you can access for free http://science.sciencemag.org/content/369/6499/65\#BIBL

http://www.sciencemag.org/help/reprints-and-permissions

Science (print ISSN 0036-8075; online ISSN 1095-9203) is published by the American Association for the Advancement of Science, 1200 New York Avenue NW, Washington, DC 20005. The title Science is a registered trademark of AAAS.

Copyright (C) 2020, American Association for the Advancement of Science 